\title{
Análisis estructural del sector turístico del Municipio Carirubana, Estado Falcón, Venezuela
}

\section{Structural analysis of the tourism sector of Carirubana Municipality, Falcón State, Venezuela}

\author{
Milagros del Carmen Gutiérrez \\ Universidad del Zulia, Venezuela \\ Mercy Narváez \\ Universidad del Zulia, Venezuela
}

Autor para correspondencia: milagros_gutierrez@hotmail.com

Fecha de recepción: 25 de Octubre de 2017 - Fecha de aceptación: 15 de Mayo de 2018

Resumen: Este artículo expone los resultados del estudio que tuvo como objetivo aplicar el método de análisis estructural, para la descripción de las relaciones que se producen entre los grupos de actores que conforman el sector turístico del municipio Carirubana, Estado Falcón, Venezuela. La investigación se desarrolló siguiendo el enfoque constructivista, caracterizándose según su tipo como descriptiva, con un diseño de campo, transeccional y no experimental. Se empleó la entrevista como técnica de investigación y como instrumento un cuestionario estructurado, el cual se aplicó a doce (12) organizaciones turísticas representativas de diferentes subsectores. La información recopilada fue procesada a través del Programa Ucinet 6, generándose la representación gráfica de la estructura social conformada por los actores y la información que dio lugar al análisis de las propiedades de conectividad, intensidad relacional y centralidad en la red de relaciones. Los resultados obtenidos dan cuenta de la presencia de relaciones sociales estrechas y descentralizadas, caracterizadas por la predominancia de vínculos fuertes, con flujos recíprocos de recursos materiales e inmateriales, destacando como actores más influyentes la alcaldía del municipio Carirubana y la Corporación para el Fomento de la Inversión Turística en la Península de Paraguaná.

Palabras Claves: análisis estructural; propiedades; red; sector turístico; ucinet 6

Abstract: This article exposes the results of the study that proposed as a goal to apply the structural analysis method, for the description of the relations that take place between the groups of actors that integrate the tourist sector of the Carirubana municipality, Falcón State, Venezuela. The research was developed following the constructivist approach, characterizing itself according to its type as descriptive, with a field, transectional and non-experimental design. The interview was used as a research technique and as a tool, a structured questionnaire, which was applied to twelve (12) representative tourism organizations from different subsectors. The information collected was processed through the Ucinet 6 Program, generating the graphic representation of the social structure formed by the actors and the information that led to the analysis of connectivity, relational intensity and centrality properties in the relationships network. The results obtained indicates the presence of close and decentralized social relations, characterized by the predominance of strong links, with reciprocal flows of material and immaterial resources, highlighting as mayor influences the mayor of the Carirubana municipality and the Corporation for the Promotion of the Tourist Investment in the Paraguaná Peninsula.

Key Words: structural analysis; properties; network; tourism sector; ucinet 6 


\section{Introducción}

En las últimas décadas, el turismo ha adquirido una creciente importancia como actividad económica y social viabilizadora del desarrollo de las zonas con potencialidades para atraer los flujos de visitantes. De allí que son cada día más notables los esfuerzos de los territorios por mejorar su competitividad en este ámbito, con miras a posicionarse en los mercados emisores nacionales e internacionales. Estos esfuerzos incluyen acciones como la puesta en valor de los atractivos (naturales y creados), la inversión en infraestructura, la actualización de los procesos de gestión y el fortalecimiento del capital social entre los diferentes grupos de actores que conforman el sector turístico.

La importancia de este último aspecto es puesta de manifiesto por Putnam (2011, p. 18), al señalar que la prosperidad de las regiones está estrechamente ligada a su capital social, incluso más que al capital económico y humano. En concordancia con este autor, Merinero (2008, p. 73) resalta que para el desarrollo turístico territorial es necesario centrar los esfuerzos y el interés analítico en fortalecer el capital social; es decir, en mejorar la capacidad de los diferentes actores que intervienen en la actividad productiva turística, para resolver de forma conjunta, problemas comunes, a través de la colaboración y la cooperación.

Sin embargo, para lograr el citado fortalecimiento, se requiere, en primer lugar, identificar plenamente a los grupos de actores que integran el sector turístico territorial, y en segundo lugar, determinar cuáles son las principales características de sus relaciones en términos de conectividad, intensidad relacional y centralidad. Para esto, resulta de gran utilidad el análisis estructural o análisis de redes sociales, descrito por Requena (2012: 5) como el enfoque que permite estudiar las estructuras sociales, a partir de los vínculos que unen tanto a miembros individuales como colectivos de la sociedad. Su operatividad se centra en tres dimensiones: la identificación de los subgrupos de actores en la estructura social, la naturaleza de las relaciones entre los actores y el efecto de la posición de los actores en la estructura.

En este marco de referencia, se presenta este artículo, en el que se exponen los principales aspectos del estudio que tuvo como objetivo central, aplicar el método de análisis estructural, para la descripción de las relaciones que se producen entre los grupos de actores que conforman el sector turístico del municipio Carirubana, Estado Falcón, Venezuela; con lo que se pretende generar conocimientos que sustenten futuras estrategias y acciones orientadas al fortalecimiento del capital social de este territorio, el cual constituye uno de los principales destinos turísticos del país.

\section{Metodología}

La investigación se desarrolló siguiendo un enfoque constructivista, definiéndose según su tipo como descriptiva, ya que la información obtenida de la misma, permitió explicar las características de las relaciones que se producen entre las organizaciones del sector turístico del municipio Carirubana. Citando a Namakforoosh (2005) "la investigación descriptiva es una forma de estudio para saber quién, dónde, cómo, cuándo y por qué del sujeto de estudio.... Se usa un diseño descriptivo para hacer una investigación, cuando el objetivo es describir las características de ciertos grupos" (p. 91). Asimismo, el estudio se sustentó en un diseño de campo, transaccional y no experimental, en virtud de que los datos se recogieron directamente de 
la realidad, durante un único lapso de tiempo; sin realizarse manipulación de variable alguna (Arias, 2012, p. 31).

Se empleó la entrevista como técnica de investigación y como instrumento el cuestionario estructurado, el cual fue sometido a validación mediante el método de juicio de expertos y posteriormente aplicado a un (1) representante de cada grupo de actores turísticos, seleccionado mediante el método de azar simple. Dicho instrumento consta de dos (2) apartados, el primero de ellos dirigido a la identificación de las organizaciones y el segundo orientado a la obtención de la información necesaria para caracterizar las relaciones entre los actores turísticos, en correspondencia con su conectividad, intensidad y centralidad.

La información recopilada fue procesada a través del software UCINET 6, el cual se utiliza específicamente para el análisis de estructuras sociales, ya que permite determinar las interacciones entre cualquier clase de individuos, representarlas mediante nodos e interconexiones y precisar sus principales propiedades. Por último, se analizó la información desde el punto de vista social, con apoyo en la revisión documental.

\section{Caracterización del territorio objeto de investigación}

Ubicada en Venezuela, al norte del Estado Falcón y bordeada de costas, la Península de Paraguaná limita al norte y al este con el Mar Caribe, al oeste con el golfo de Venezuela y al sur con el golfete de Coro y el municipio Miranda del Estado Falcón. La integran tres Municipios: Los Taques, Falcón y Carirubana. Posee una superficie de 3.405 km2 y 327.238 habitantes, según el Instituto Nacional de Estadística (INE, 2014); de los cuales, el 73, 17\% se ubica en el municipio Carirubana.

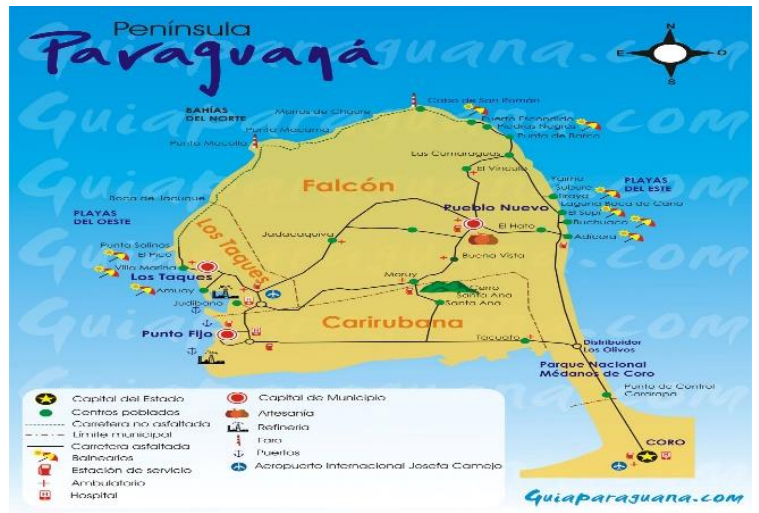

Figura 1. Mapa político - turístico de la Península de Paraguaná. Fuente:http://blogeducativodelturismo.blogspot.com/2015

Constituye una de las zonas más cálidas de Venezuela, ubicada frente al Mar Caribe, cerca de las islas de Aruba, Curazao y Bonaire. Cuenta con un medio climático semiárido, gran diversidad natural, hermosas playas, variedad de manifestaciones religiosas y culturales, y sitios de interés histórico y comercial. Todo esto potencia a la Península como la puerta de entrada por excelencia para el turismo internacional; constituyendo también un importante destino nacional, de fácil acceso para los residentes en la región centro occidental del país. 
En cuanto a su economía, hasta 1945 la misma dependió de la siembra y comercialización de sábila, así como de la cría de ganado caprino y ovino; no obstante, a partir de esa fecha comenzó a desarrollarse la industria petrolera. Por otra parte, en 1974 la Península fue declarada Zona de Utilidad Pública y de Interés Turístico Recreacional. Con esta declaración se sentaron las bases para el auge turístico que se inició en 1996, cuando la necesidad de diversificar la economía, desarrollando el turismo como complemento de la actividad petrolera, llevó a la Fundación para el Desarrollo de la Región Centro Occidental de Venezuela (FUDECO), a diseñar ocho (8) productos turísticos para ser ofrecidos en el territorio. Posteriormente, en el año 1998, se creó la Ley de Zona Libre para el Fomento de la Inversión Turística en la Península de Paraguaná, como Régimen Especial Territorial de carácter fiscal, que abarca el área geográfica comprendida por los territorios de los Municipios Carirubana, Falcón y los Taques (Asamblea Nacional de la República Bolivariana de Venezuela, 1998).

Desde la implementación del mencionado régimen, se ha promovido la creación de empresas turísticas y comerciales conexas al turismo, las cuales se encuentran asentadas principalmente en el municipio Carirubana. De igual manera, a nivel municipal y estadal se han establecido organizaciones gubernamentales para contribuir con la gestión turística y se han conformado gremios empresariales. Todas estas formas de organización, en conjunto con los medios de comunicación, las comunidades receptoras y las instituciones financieras, constituyen el sector turístico del municipio y tienen como propósito, velar por su desarrollo integral y sustentable.

\section{Caracterización de los grupos de actores que conforman el sector turístico territorial}

En la literatura turística logran identificarse seis grupos de actores, cuya participación en esta actividad económica resulta indispensable para garantizar el desarrollo competitivo de los destinos, mediante la articulación equilibrada de intereses, el establecimiento de objetivos integrales y la conducción pluridimensional de los mismos, a saber:

Sector Empresarial. Está conformado por las empresas turísticas encargadas de ofrecer los productos y servicios para ser consumidos o utilizados por los visitantes en el destino turístico. Según Guerrero y Ramos (2014, p. 108) las empresas turísticas son las dedicadas específicamente a actividades de alojamiento, restauración, transporte turístico, cultura y recreación, oficinas de información y agencias de viajes. Según Eiquez (1988, p. 11), en este sector también se pueden ubicar los gremios empresariales, como organizaciones que agrupan y representan los intereses colectivos de las empresas o personas cuyas actividades pertenecen a la rama turística

Estado. Interviene en la materia turística a través de las entidades gubernamentales con competencia en turismo, desarrollando actividades orientadas a identificar las prioridades del territorio en el ámbito turístico, decidir cuántos y cuáles recursos se dedican al turismo, en términos económicos, tecnológicos y humanos; coordinar los objetivos de los diferentes actores turísticos, y representar los intereses de los mismos, propiciando estructuras de interacción coordinadas y bien organizadas (Velasco, 2016, p. 579). 
Instituciones de Formación Turística. Su función principal consiste en mejorar la cualificación del recurso humano que presta servicios en el sector turístico, promoviendo su crecimiento profesional y la adquisición de conocimientos sobre nuevas tecnologías, especialidades y lenguas extranjeras (Rivas, 2014, p. 51), a través del desarrollo y la gestión de programas de formación.

Instituciones Financieras. A través del aporte de recursos financieros, estas instituciones contribuyen a aumentar la oferta de bienes y servicios en el mercado, amplían la capacidad de producción de las empresas y contribuyen con el desarrollo de las actividades industriales, comerciales y de servicios. De igual manera, las entidades financieras destinan importantes porcentajes de recursos para desarrollar, en conjunto con el Estado, las áreas prioritarias para la economía nacional; entre ellas, la turística (Linares, 2013, p. 223).

Medios de Comunicación. Llevan a cabo acciones de promoción y difusión, dando a conocer los mercados y productos turísticos, y facilitando a los visitantes la organización del viaje desde su lugar de origen. En este sentido, Altamirano y Túnez (2014, p. 2) destacan la importancia de las nuevas tecnologías de la información y la comunicación (TIC's), como herramientas que han permitido en los últimos años promocionar a nivel internacional las ventajas competitivas de los destinos turísticos, lo que ha generado cambios favorables en los mismos, reflejados en su desarrollo social, crecimiento económico, imagen y posicionamiento.

Comunidad Receptora. Siguiendo a Monterrubio (2009, p.104), la comunidad receptora se presenta como el conjunto conformado por un espacio geográfico y sus habitantes, los cuales comparten valores, creencias y actitudes, y poseen características comunes, en cuanto a tradiciones, gastronomía, vestido e idioma. Al ser un elemento constante en los territorios, las comunidades desempeñan una función importante en la conservación de los atractivos y la infraestructura, e influyen a través de su apertura en la percepción de los turistas sobre el destino. Tomando la información previamente expuesta como referencia, en el cuadro 1 se identifican los actores turísticos del municipio Carirubana que participaron en el estudio:

Sector Empresarial

Gremios

Empresariales

Organizaciones

Estatales

Instituciones de Formación Turística

$\square$ Instituciones

Bancarias

Medios de

Comunicación
- Una (1) agencia de viajes.

- Una (1) empresa de alimentos y bebidas.

- Un (1) empresa de alojamiento.

- Cámara de Turismo de Paraguaná.

- Instituto Nacional de Turismo INATUR (nivel Nacional)

- Corporación Falconiana de turismo CORFALTUR (nivel Estadal)

- Corporación para el Fomento de la Zona Libre de Inversión Turística de la

Península de Paraguaná CORPOTULIPA (Nivel Regional-Península de Paraguaná).

- Dirección de Turismo de la Alcaldía del municipio Carirubana (nivel Municipal).

- Una (1) Universidad que imparte la carrera de Turismo.

- Una (1) Entidad financiera de carácter privado.

- Un (1) diario impreso de circulación regional. 
Comunidad

- Un (1) Consejo Comunal con vocación turística.

Receptora

Fuente: Elaboración propia (2017)

Cuadro 1. Organizaciones del sector turístico que participaron en el estudio

\section{Descripción del método de análisis estructural}

El Análisis Estructural, también conocido como Análisis de Redes Sociales, es una metodología que se emplea para el análisis formal de las redes de relaciones que tienen lugar entre actores, partiendo de preguntas básicas sobre sus interacciones (Betancourt y Murcia, 2017, p. 2). Para comprender esta metodología, Wasserman y Faust (2013, p. 36) subrayan la importancia de tomar en cuenta los siguientes aspectos: a) los actores y sus acciones se consideran unidades interdependientes más que independientes y autónomas, y b) los lazos (vínculos) relacionales entre los actores son canales de transferencia o flujo de recursos materiales e inmateriales.

De lo expuesto se colige que existen dos elementos básicos, a partir de los cuales se puede analizar la estructura de los grupos sociales o redes: los nodos, concebidos como agentes o actores sociales, y los vínculos o interconexiones, los cuales representan las relaciones de intercambio o de cooperación que se establecen entre los actores, señalando cuáles son los diferentes caminos que puede tomar el flujo de recursos. Así, el Análisis Estructural parte de ambos elementos para estudiar las siguientes propiedades:

Conectividad. Analiza el contenido, la direccionalidad y fortaleza de los vínculos entre los actores que forman parte de una estructura social. Con respecto al contenido, éste puede referirse a recursos materiales o inmateriales (información, bienes, servicios). Por su parte, la direccionalidad, estudia las conexiones recíprocas o en un solo sentido que pueden tener lugar entre los actores; en este sentido, exponen Mendieta y Schimdt (2002, p. 7) que en las conexiones recíprocas los recursos fluyen en sentido de ida y vuelta; es decir lo que un actor aporta a otro es retornado por este último en igual o diferente especie, en el corto, mediano o largo plazo. Por otro lado, las conexiones en un solo sentido se presentan cuando los recursos fluyen desde un actor a otro, sin ser retornados por este último.

Por último, la fortaleza de los vínculos puede ser determinada a partir de diferentes criterios, siendo los más utilizados, la multiplicidad de recursos transferidos a través de las conexiones, el tiempo dedicado y los servicios recíprocos que caracterizan las relaciones (Granovetter, 1973, p. 1361). También se utilizan como medida de la fortaleza de los vínculos la intimidad (reflejada en la proximidad o intensidad emocional entre los actores) y la frecuencia de contactos, referida por Brown y Conrad (2001, p. 443) como el número de veces que un actor mantiene contacto con otro.

Intensidad relacional. Proporciona información acerca de la cantidad total de conexiones establecidas en el grupo social, así como sobre el número de vínculos de cada uno de los actores, a partir de tres medidas:

- Densidad. Ofrece información sobre la alta o baja conectividad en la estructura social. Representa el porcentaje de conexiones existentes, con respecto al máximo de conexiones 
posibles, indicando así la intensidad de las relaciones en el conjunto de toda la red (Merinero y Pulido, 2009, p. 181).

- Grado nodal. Se refiere a la cantidad de vínculos directos (conexiones) que tiene cada actor en la red. Esta medida proporciona información sobre la posición que ocupan los actores dentro de la estructura social, de la que se deriva su posibilidad y capacidad de acción. En este sentido, Lozares (1996, p. 122) plantea que cuantas más relaciones se mantienen, más acceso se tiene a los recursos sociales.

- Densidad relacional del nodo. Se refiere a la relación entre el número de conexiones establecidas por un actor, y el número máximo de conexiones posibles que este actor puede tener, expresada en términos relativos (Garrido, 2001, p. 9). En otras palabras, indica la proporción de las posibles conexiones de cada actor que, de hecho, están presentes.

Centralidad. Un actor se considera central en la medida que se encuentra implicado en todas las relaciones o asume un rol de intermediación entre otros actores. Se admiten, por tanto, dos principales criterios para medir la centralidad, el primero de ellos es el grado nodal y el segundo la intermediación. Cuanto mayor sea la centralidad de un actor, mayor será su capacidad para incidir en la opinión y conducta de otros, especialmente en los casos de flujos de información e intercambio (Garrido, 2001, p. 9).

Otro criterio valioso para medir la centralidad tiene que ver con la proximidad, medida a partir de la cantidad de intermediarios que necesita cada actor para llegar a los demás. Al respecto, Lozares, López, Bolíbar y Muntanyola (2013, p.85), señalan que esta medida tiene que ver con la capacidad de llegar a todos los actores o, en todo caso, de llegar al máximo de actores posible con el mínimo número de intermediarios. En consonancia con estos autores, se considera la proximidad como un importante recurso relacional asociado a la posibilidad de comunicarse, ayudar y apoyar, que permite reducir el dispendio de recursos económicos y de tiempo.

\section{Análisis estructural del sector turístico del Municipio Carirubana, Península de Paraguaná.}

En la figura 2 se presenta la matriz contentiva de la información sobre los datos relacionales entre los actores turísticos del municipio Carirubana, la cual fue recopilada mediante la aplicación del instrumento de investigación a sus principales representantes (propietarios, presidentes, coordinadores). Se trata de una matriz cuadrada, con igual número de filas y columnas (12), en la que para cada pareja de actores se identificó, primeramente, la existencia o no de una relación y la fortaleza de los vínculos, con base en el criterio de frecuencia anual de contactos. Para tal fin, se propuso el siguiente baremo:

\begin{tabular}{ccc}
\hline Frecuencia anual de contactos & Fortaleza de los vínculos & Número asignado en la matriz \\
\hline $\mathbf{0}$ & No existe relación & $\mathbf{0}$ \\
$\mathbf{1}$ & Baja & 1 \\
$\mathbf{2}$ a 10 & Media & 2 \\
\hline
\end{tabular}




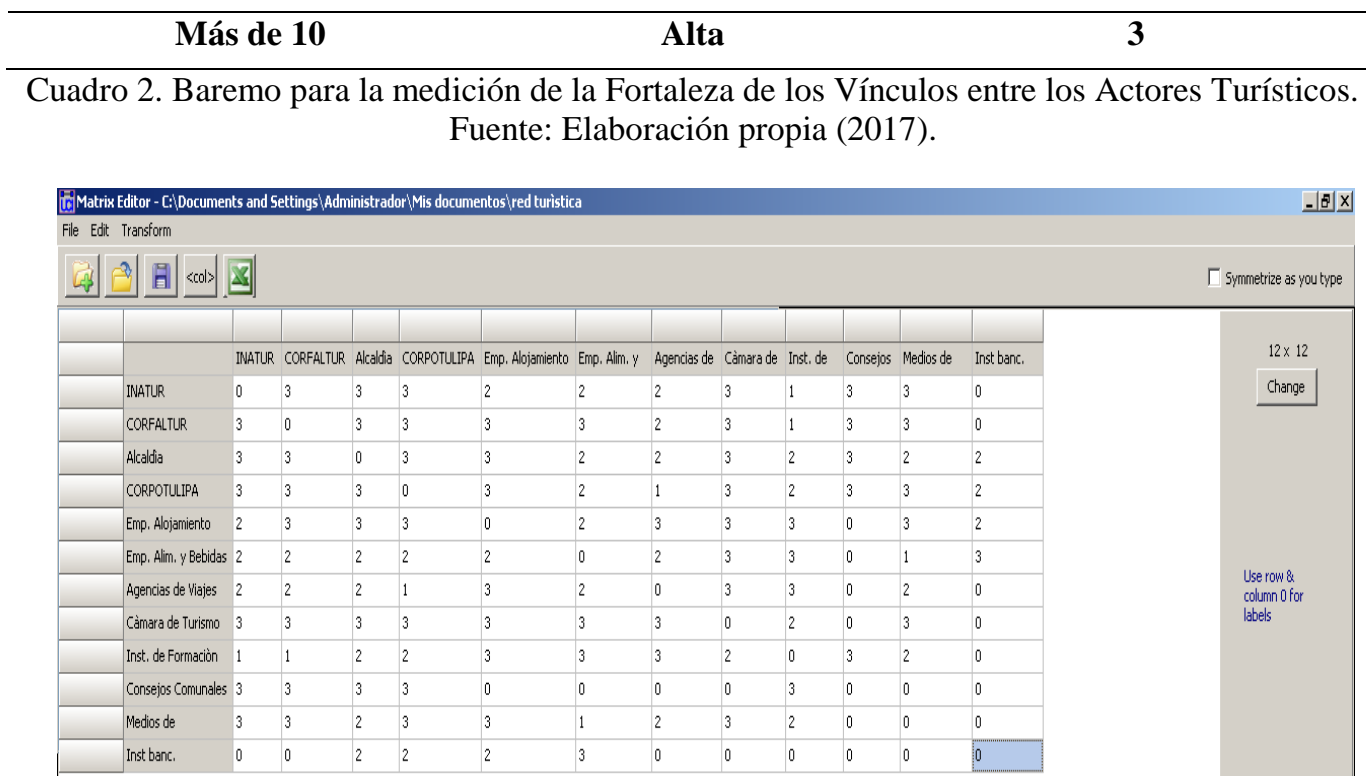

Figura 2. Matriz de Relaciones entre los Actores Turísticos del Municipio Carirubana Fuente: Elaboración propia (2017), a través del Programa UCINET6.

Luego de introducir en la matriz los datos correspondientes a las interacciones, se generó el gráfico reticular (socio grama) representativo de las mismas, mediante la aplicación Netdraw, adjunta al programa UCINET 6 (Figura 3); además, a partir de estos datos se calcularon las propiedades de las relaciones.

\section{Representación Gráfica de la Red de Actores Turísticos}

En la figura 3 se muestra la red de relaciones existente entre los doce (12) actores turísticos del municipio Carirubana, conectados a través de 54 flechas bidireccionales. El color de los nodos permite ubicar a los actores en cada uno de los grupos establecidos en el cuadro 1; además, la fortaleza de los vínculos entre cada pareja de actores se identifica en las conexiones.

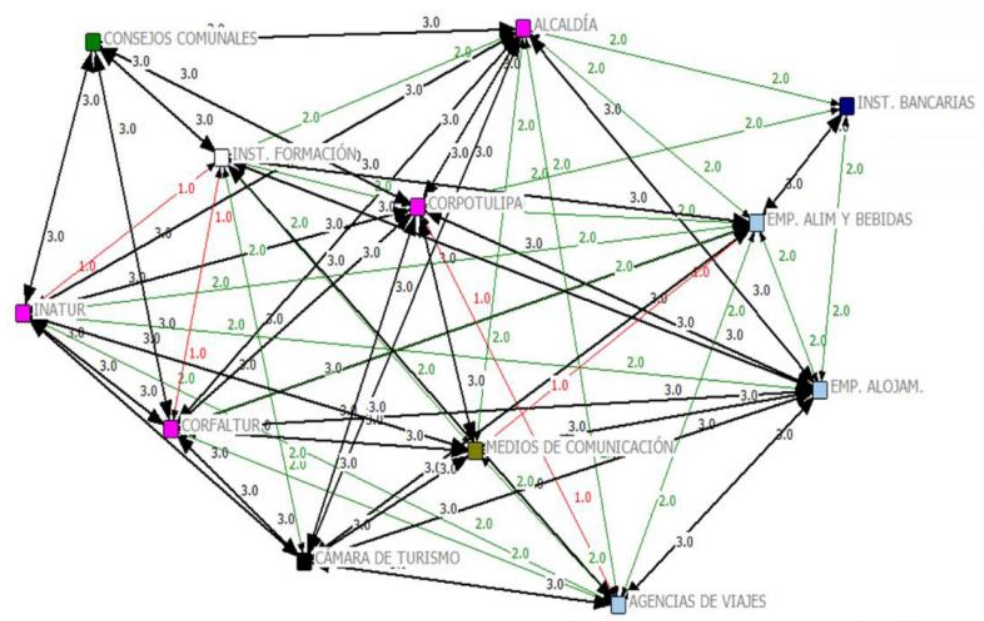

Figura 3. Representación gráfica de la red de relaciones entre los actores turísticos del municipio Carirubana.

Fuente: Elaboración propia (2017), a través del Programa UCINET 6.

Análisis de las propiedades de la red. 


\section{Conectividad.}

- Con respecto al contenido de las relaciones, los actores refirieron el intercambio de recursos materiales (equipos, insumos, recursos económicos) e inmateriales (información, asesorías, servicios) entre ellos. En cuanto a la direccionalidad de los vínculos, los resultados obtenidos de la aplicación del instrumento indican que las relaciones entre los actores son recíprocas, por tanto los flujos son bidireccionales (todos los participantes señalaron que los recursos que aportan a otro actor, son devueltos por éste último, en igual o diferente especie). Por otro lado, del análisis de la fortaleza de las conexiones, se colige que entre las organizaciones predominan los vínculos fuertes (32 de 54).

Intensidad Relacional.

- Densidad de la Red. En la figura 4, correspondiente a la impresión de pantalla de los datos obtenidos a través del programa Ucinet 6 , luego de procesar la información presentada en la matriz de relaciones (figura 2), se muestra que la densidad de la red analizada es 0.818 , lo cual indica que se trata de una estructura social densa en la que se producen el $81,8 \%$ de todas las relaciones posibles.

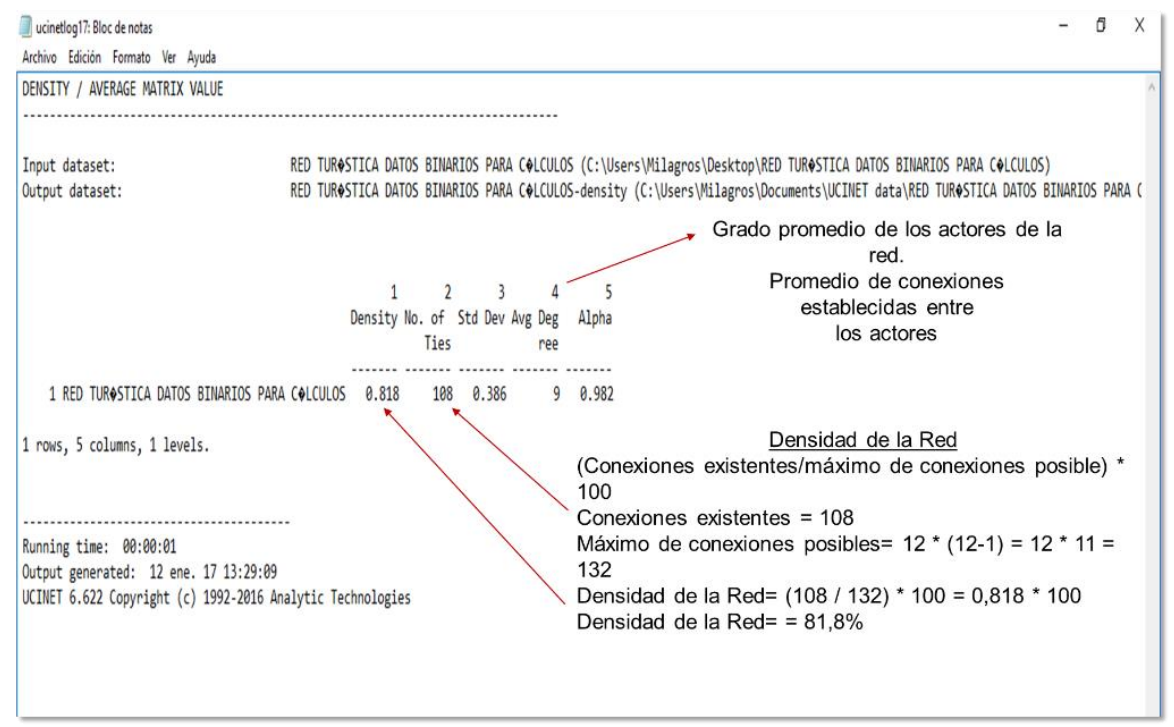

Figura 4. Densidad de la Red

Fuente: Elaboración propia (2017), a través de las aplicaciones del Programa UCINET 6.

Al respecto, es importante acotar que las redes sociales densas estimulan la cooperación, fomentan el desarrollo de normas sólidas de reciprocidad; facilitan la comunicación y mejoran el flujo de información; además, incrementan el costo potencial para los actores que no se comporten de manera confiable (al poner en juego la propia reputación) y representan el éxito que la colaboración ha tenido en el pasado, lo que podría funcionar como patrón culturalmente definido para la colaboración futura (Forni, Siles y Barreiro, 2004, p. 4).

- Grado nodal y densidad relacional de los nodos (actores turísticos). De acuerdo con la información presentada en la Figura 5, puede afirmarse que los actores 
turísticos (nodos) con mayor grado y densidad relacional son la alcaldía del municipio Carirubana y la Corporación para la Zona Libre para el Fomento de la Inversión Turística en la Península de Paraguaná (CORPOTULIPA). Ambas entidades gubernamentales son, por tanto, las más influyentes y las que tienen mayor capacidad de acceso a la información en la red (centrales, en función del grado nodal). Por otro lado, los actores con menor cantidad de conexiones y menor densidad relacional son los Consejos Comunales (en representación de las comunidades receptoras) y las Instituciones Bancarias.

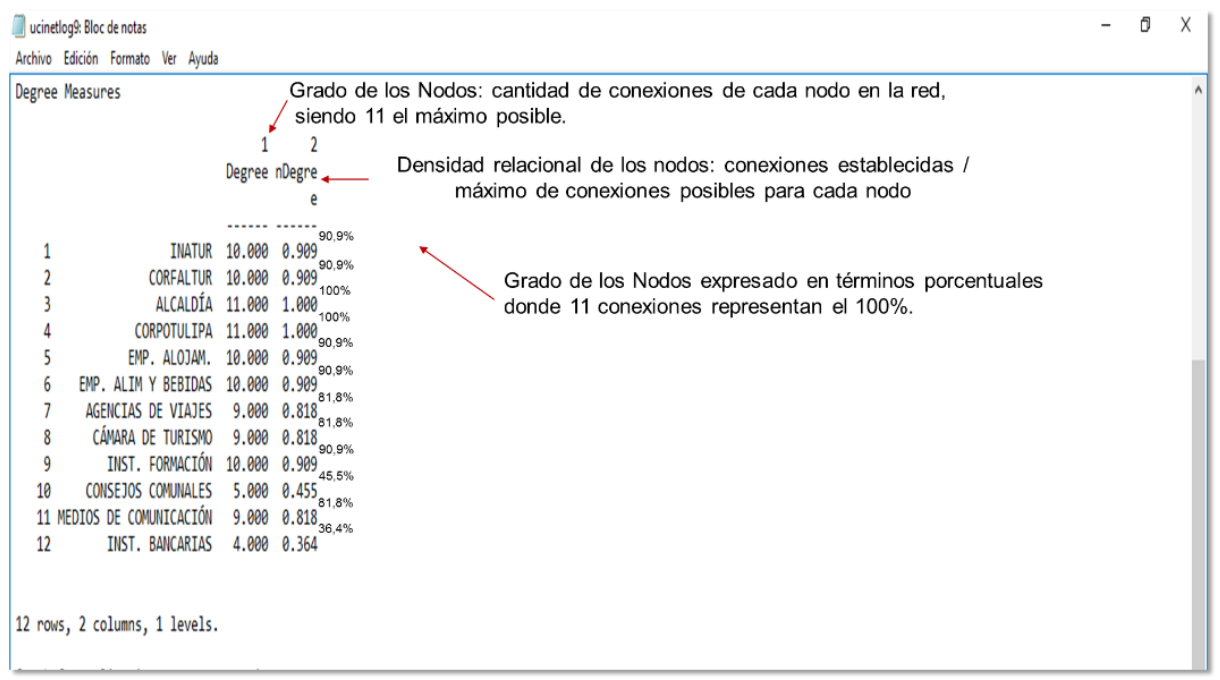

Figura 5. Grado Nodal y Densidad Relacional de los nodos (Actores Turísticos) Fuente: Elaboración propia (2017), a través de las aplicaciones del Programa UCINET 6.

\section{Centralidad.}

- Grado de intermediación de los actores e índice de centralización de la red. En la Figura 6 se presentan los resultados generados por el software Ucinet 6 sobre el grado de intermediación que tienen los actores turísticos locales cuyas interacciones se representan en la red, tomando como base todos los posibles caminos de vínculos que conectan a todos los pares posibles de actores. En este sentido, pudo evidenciarse que las organizaciones con mayor grado de intermediación son la Corporación para la Zona Libre para el Fomento de la Inversión Turística en la Península de Paraguaná y la alcaldía del municipio Carirubana (aparecen 3 veces en los caminos que conectan a otros pares de actores). 


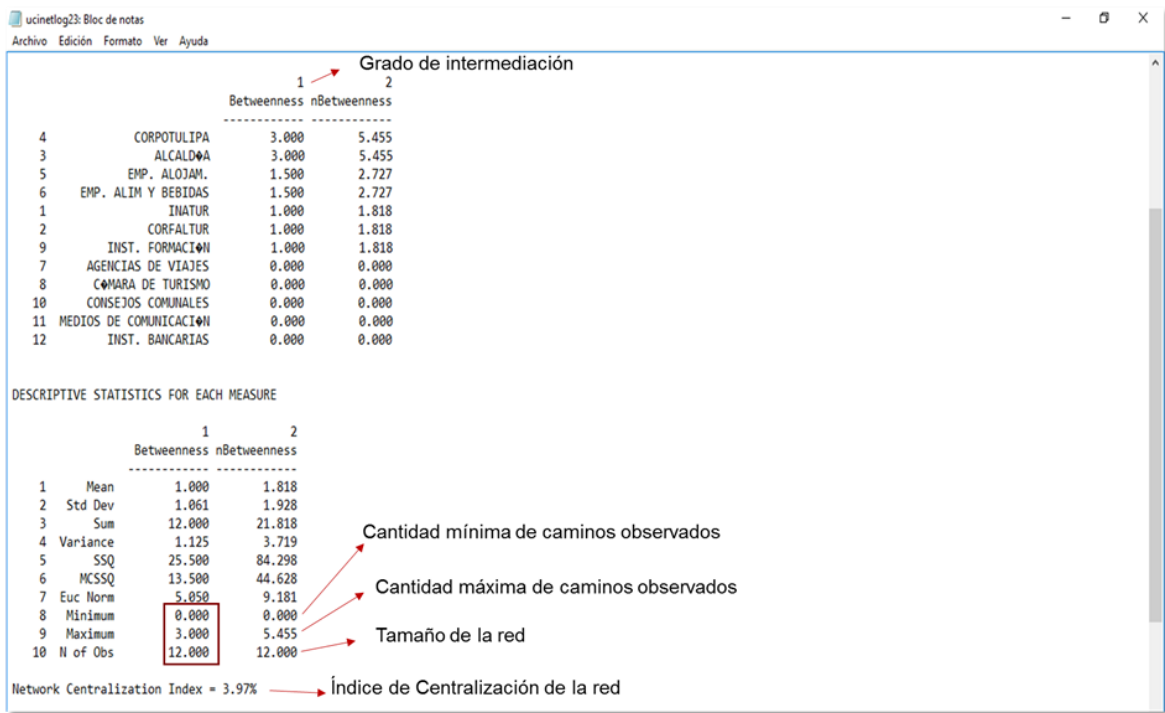

Figura 6. Grado de Intermediación de los Actores e Índice de Centralización de la Red. Fuente: Elaboración propia (2017), a través de las aplicaciones del Programa UCINET 6.

En cuanto al Índice de Centralización general de la red (presentado en la parte inferior de la figura 6), esta medida se refiere al grado en que la red se concentra alrededor de un actor específico, el cual controla todas las relaciones. Cuanto más se acerque este indicador al 100\%, mayor será la tendencia de la red a concentrase alrededor de un actor; por el contrario, valores bajos en este índice son señal de una estructura descentralizada (Velázquez y Aguilar, 2005, p. 24). La información derivada del programa UCINET 6, indica que la centralización de la red de actores turísticos es de $3,97 \%$, por tanto, se trata de una estructura descentralizada, de la que puede inferirse que aun cuando es evidente la presencia de actores destacados por su capacidad de intermediación y poder, éstos no controlan completamente las relaciones.

Proximidad.

- Esta medida toma como referencia la cantidad de intermediarios que necesita cada actor para llegar a todos los demás. Cuantos menos intermediarios utilice un actor, mayor será su cercanía al resto. Así, con base en el análisis de los datos generados por el Programa UCINET 6, presentados en la figura 7, puede afirmarse que la alcaldía de Carirubana y Corpotulipa son los únicos actores que no necesitan intermediarios para llegar a todos los participantes en la red, puesto que se vinculan a través de 11 conexiones con los 11 actores restantes; en otras palabras, emplean una (1) sola conexión para llegar a cada actor. 


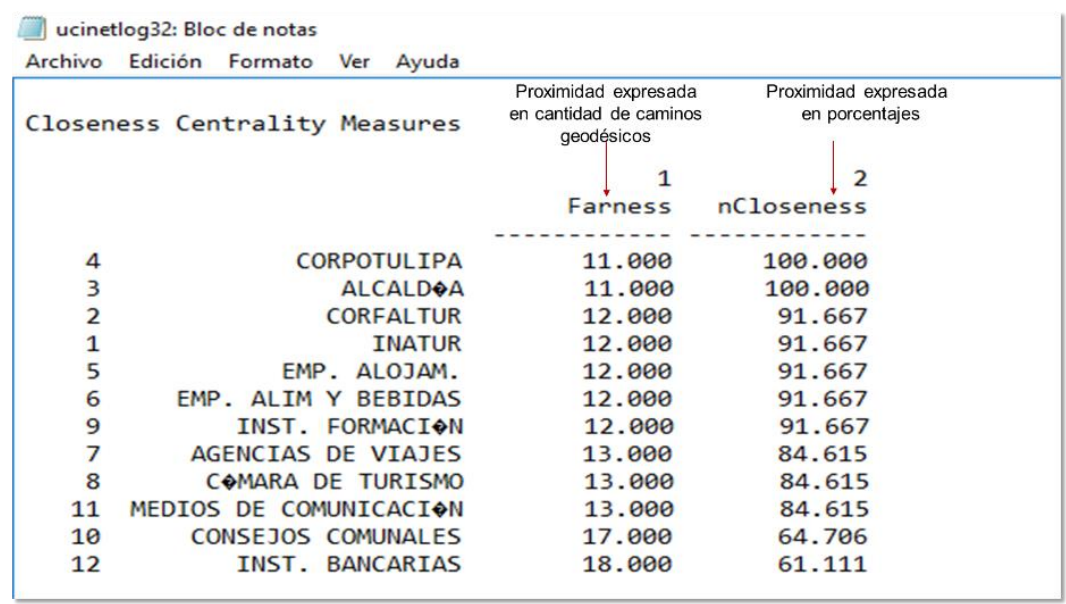

Figura 7. Proximidad de los Actores

Fuente: Elaboración propia (2017), a través de las aplicaciones del Programa UCINET 6.

\section{Conclusiones}

A la luz del objetivo planteado para el estudio, el cual consistió en aplicar el método de análisis estructural, para la descripción de las relaciones que se producen entre los grupos de actores que conforman el sector turístico del municipio Carirubana, Estado Falcón, Venezuela, pueden extraerse las siguientes conclusiones:

Existen seis grupos de actores que, integrados, conforman el sector turístico territorial; a saber: las empresas y gremios empresariales, el Estado, las instituciones de formación turística, las instituciones financieras, los medios de comunicación y las comunidades receptoras. Las relaciones que se producen entre ellos son importantes para promover el desarrollo competitivo de los destinos turísticos, de allí la necesidad de estudiarlas, con el propósito de obtener conocimientos que sustenten estrategias y acciones orientadas a fortalecerlas.

Para estudiar las relaciones que tienen lugar entre los mencionados actores, se emplea el Análisis Estructural. Esta metodología, además de ofrecer la posibilidad de visualizar gráficamente las relaciones, las analiza en relación con tres (3) propiedades básicas: la conectividad, referida al contenido, direccionalidad y fortaleza de los vínculos; la intensidad relacional, que proporciona información acerca de la cantidad de conexiones establecidas en la red y sobre el número de vínculos de cada actor; y la centralidad de los actores, medida a partir del grado nodal, el grado de intermediación y la proximidad.

Al respecto de la conectividad, la información recopilada a partir del instrumento de recolección de datos indica que entre los actores predominan los vínculos fuertes, fluyen a través de los mismos, de manera bidireccional (recíproca), bienes, insumos, recursos económicos, servicios, asesorías e información. En cuanto a la intensidad relacional, del estudio se desprende que en la estructura social se producen el $81,8 \%$ de todas las relaciones posibles; asimismo, puede afirmarse que los actores turísticos con mayor grado y densidad relacional son la alcaldía del municipio Carirubana y la Corporación para la Zona Libre para el Fomento de la Inversión Turística en la Península de Paraguaná. Esto les otorga una posición de prestigio, influencia y 
control en la estructura social. Estos actores se presentan también como los más centrales en términos de conexiones directas, intermediación y proximidad.

Por lo antes expuesto, se considera que las estrategias que sean propuestas para fortalecer las relaciones entre los actores turísticos del municipio Carirubana, deben dirigirse a incrementar la participación de las comunidades, entidades financieras y empresas en las relaciones y en los procesos de gestión turística. Además, el poder de mediación y acceso a otros actores que tienen la alcaldía y la Corporación para la Zona Libre para el Fomento de la Inversión Turística en la Península de Paraguaná, puede ser aprovechado como mecanismo para convocar, y ejercer influencia sobre los actores sociales.

\section{Bibliografía}

Altamirano, V. y Túnez, M. (2014). Promoción y difusión turística en Iberoamérica. Análisis de contenidos y herramientas utilizadas en portales Web y redes sociales. VI Congreso Internacional Latino de Comunicación Social. $\quad$ España. Obtenido de: http://www.revistalatinacs.org/14SLCS/2014_actas/050_Altamirano.pdf

Arias, F. (2012). El Proyecto de Investigación. Caracas: Episteme.

Asamblea Nacional de la República Bolivariana de Venezuela (1998) Ley de Creación y de Régimen de la Zona Libre para el Fomento de la Inversión Turística en la Península de Paraguaná, Estado Falcón. Caracas, Venezuela. Gaceta Oficial Nº 36.517.

Betancourt, X. y Murcia, J. (2017). El Análisis de Redes Sociales -ARS- como recurso metodológico para el estudio formal de redes de políticas públicas: Congreso latinoamericano de Ciencia Política. Montevideo, Uruguay.

Brown, D. y Conrad, A. (2001). Granovetter was Right. The Importance of Weak Ties to a Contemporary Job Research. Group Organization Management. $\mathrm{N}^{\circ}$ 26, Pp. 434 - 462. Obtenido de: http://gom.sagepub.com/content/26/4/434.short

Eiquez, E. (1988). La Organización del Sector Empresarial Privado. Revista sobre Relaciones Industriales y Laborales. Obtenido de: file:///C:/Documents\%20and\%20Settings/Administrador/Mis\%20documentos/Downloads/15644934-1-PB.pdf

Forni, P., Siles, M. y Barreiro, L. (2004). ¿Qué es el Capital Social y Cómo Analizarlo en Contextos de Exclusión Social y Pobreza? Research Report, 35. Pp. 1-15. Obtenido de: https://jsri.msu.edu/upload/research-reports/rr35.pdf

Garrido, F. (2001). El Análisis de Redes en el Desarrollo Local. Universidad Complutense de Madrid, Facultad de Ciencias Políticas y sociología. Obtenido de: http://www.redcimas.org/wordpress/wpcontent/uploads/2014/02/m_JGarrido_ANALISISdeREDES.pdf

Granovetter, M. (1973). The Strenght of Weak Ties. American Journal of Sociology, 78 (6), 1360 - 1380. Obtenido de: https://sociology.stanford.edu/sites/default/files/publications/the_strength_of_weak_ties_and_exc h_w-gans.pdf 
Guerrero, P. y Ramos, R. (2014). Introducción al Turismo. D.F., México: Grupo Editorial Patria.

Instituto Nacional de Estadística. (2014). XIV Censo Nacional de Población y Vivienda. Resultados por Entidad Federal y Municipio del Estado Falcón. Falcón, Venezuela. Obtenido de: http://www.ine.gov.ve/documentos/Demografia/CensodePoblacionyVivienda/pdf/falcon.pdf

Linares, H. (2013). Banca Venezolana. Caracas: Humberto Linares.

Lozares, C. (1996). La Teoría de Redes Sociales. Papers, 48, 103-126. Obtenido de: http://arsuns.blogspot.com/2013/11/lozares-la-teoria-de-las-redes-sociales.html

Lozares, C., López, P., Bolívar, M. y Muntanyola, D. (2013). La Centralidad en las Redes Sociales: medición, correlación y aplicación. Metodología de Encuestas, 15, 77 - 97. Obtenido de: http://casus.usal.es/pkp/index.php/MdE/article/view/1038/977

Mendieta, J. y Schmidt, S. (2002). Análisis de Redes: aplicaciones en ciencias sociales. D.F., México: Instituto de Investigaciones en Matemáticas Aplicadas y en Sistemas.

Merinero, R. (2008). Micro-cluster turísticos: el papel del capital social en el desarrollo económico local. Revista de Estudios empresariales. Segunda Época. 2, 67-92. Obtenido de: file://C:/Documents\%20and\%20Settings/Administrador/Mis\%20documentos/Downloads/3661272-1-PB.pdf

Merinero, R. y Pulido, J. (2009). Desarrollo turístico y dinámica relacional. Metodología de Análisis para la Gestión Activa de Destinos turísticos. Cuadernos de turismo, 23, 173-193. Obtenido de: https://digitum.um.es/xmlui/bitstream/10201/15449/1/67561.pdf

Monterrubio, J. (2009). Comunidad receptora: Elemento esencial en la gestión turística. Gestión Turística, $\mathrm{N}^{\mathrm{o}}$ 11, pp 101-111. Obtenido de: http://www.redalyc.org/articulo.oa?id=223314808005

Namakforoosh, M. (2005). Metodología de la Investigación. D.F., México: Limusa.

Putnam, R. (2011). Para que la democracia funcione: Las tradiciones cívicas en la Italia moderna. Madrid: Cyan Proyectos Editoriales.

Requena, F. (2012). Análisis de redes sociales. Orígenes, teorías y aplicaciones. Madrid: Centro de Investigaciones Sociológicas.

Rivas, J. (2014). Planificación Turística. Asturias: Septem Ediciones.

Velasco, M. (2016). Entre el poder y la racionalidad: gobierno del turismo, política turística, planificación turística y gestión pública del turismo. PASOS, Revista de Turismo y Patrimonio cultural, Vol. 14, No. 3, 577-594. Obtenido de: http://www.redalyc.org/articulo.oa?id=88145925002

Velázquez, O., y Aguilar, N. (2005). Manual Introductorio al Análisis de Redes sociales. Revista Red-Iris, pp. 1 - 49. Obtenido de: http://revista-redes.rediris.es/webredes/talleres/Manual_ARS.pdf

Wassserman, S. y Faust, K. (2013). Análisis de Redes Sociales. Métodos y aplicaciones. Madrid: Centro de Investigaciones Sociológicas. 